\title{
NMR Relaxation Study on a Preferential Adsorption in Polymer Solution
}

\author{
Kazuo SAto and Atsuo NishiokA \\ Department of Polymer Engineering, Tokyo Institute of Technology, \\ Ookayama, Meguro-ku, Tokyo, Japan.
}

(Received July 20, 1971)

\begin{abstract}
KEY WORDS Spin-Lattice Relaxation / Preferential Adsorption / Poly(methyl methacrylate) /
\end{abstract}

In ternary solvent(1) - non solvent(2) - polymer (3) systems, phase separation, preferential and "inverse" adsorption phenomena have been observed and discussed on the basis of thermodynamic theory and measurements. ${ }^{1-3}$ In this short communication, we report a proton spinlattice relaxation study of the benzene(1)methanol(2)-poly(methyl methacrylate) (PMMA) system and discuss the anomalous relaxation behavior and the interaction between solvents and PMMA. This method is useful for studing microscopic interactions because the spin-lattice relaxation is sensitive to the intermolecular interactions and the relaxation times can be measured separately on chemically shifted protons.

The viscosity-average molecular weight and the tacticity of samples studied were $41 \times 10^{4}$ and $100 \%$ for isotactic PMMA, and $10^{5}$ and $83 \%$ for syndiotactic PMMA, respectively. Measurements of the spin-lattice relaxation time $T_{1}$ of the solvent molecules were carried out at $25 \pm 1^{\circ} \mathrm{C}$ by the adiabatic rapid-passage method with sampling using a JNMC-60 H spectrometer operated at $60 \mathrm{MHz}$.

In Figure 1, $T_{1}$ values in benzene-methanol mixed solvent are plotted against mole fraction of methanol. $T_{1}$ of benzene is almost constant, whereas $T_{1} \mathrm{~s}$ of the methyl and the hydroxyl protons decrease with increasing mole fraction of methanol. This is believed to be due to the effect of the self-association of methanol, which would reduce the molecular motions. Figure 1 presents plots of $T_{1}$ for benzene and methanol in the presence of PMMA at a concentration of $5 \mathrm{~g}$ per $100-\mathrm{m} l$ solvent. A maximum of $T_{1}$

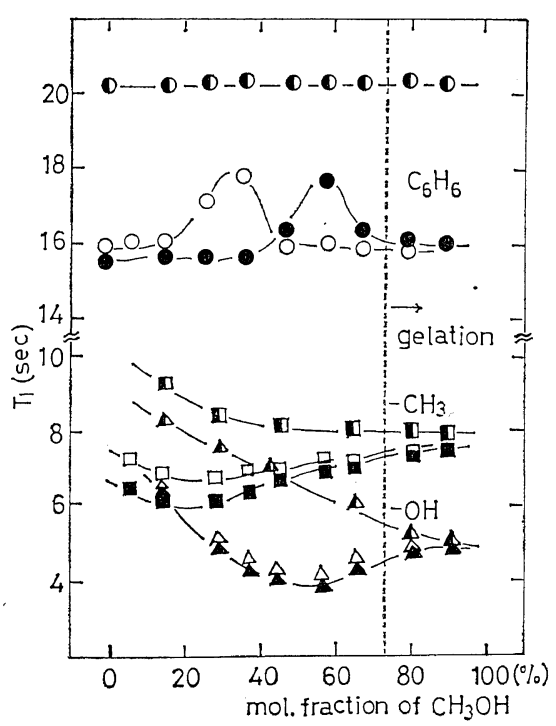

Figure 1. $T_{1}$ vs. mole fraction of methanol. Circles, squares, and triangle represent the $T_{1}$ values of the benzene methyl and hydroxyl protons, respectively: $\boldsymbol{D}, \boldsymbol{\Delta}$, and $\Delta$, benzene-methanol system; $\bigcirc$, and $\square$, and $\triangle$, isotactic PMMA solution; $\mathbf{0}$, and $\boldsymbol{\Delta}$, syndiotactic PMMA solution.

for benzene appears at lower concentration of methanol for isotactic PMMA than for syndiotactic PMMA. This is very different from the relaxation of benzene and chloroform in the benzene-chloroform-PMMA system, where the effect of the tacticity is very slight. ${ }^{4}$ Minima in $T_{1}$ are observed for the methyl and the hydroxyl protons, and the effect of the tacticity on the $T_{1}$, while slight, is observable. As the concentration of methanol increases beyond that 


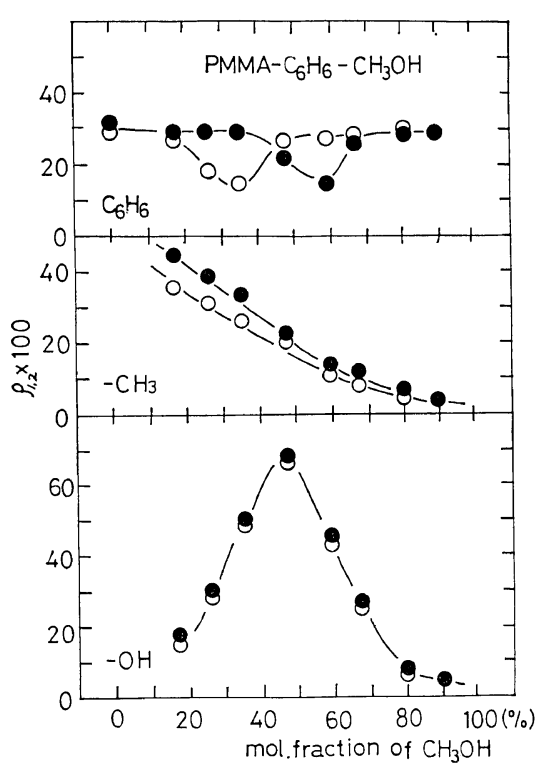

Figure 2. $\rho$ vs. mole fraction of methanol; benzene-methanol-PMMA system: $\bigcirc$, isotactic PMMA solution; , syndiotactic PMMA solution.

of the $T_{1}$ minima, the $T_{1} \mathrm{~s}$ of both types of methanol protons tend to approach their respective $T_{1} \mathrm{~s}$ in the system without PMMA. At a composition where gelation of PMMA solutions is observed the effect of the presence of PMMA is only slight. This suggests that the methanol molecules in the gelled ternary system behave in the same manner as in the mixed solvent.

In eq 1 we define a reduced relaxation rate $\rho$, which represents the relative effect of polymer molecules on the spin-lattice relaxation rote of the solvent molecules in solution.

$$
\rho=\left\{\left(T_{1 \mathrm{obs}}^{-1}\right)-\left(T_{1 \mathrm{f}}^{-1}\right)\right\} /\left(T_{1 \mathrm{f}}^{-1}\right)
$$

where $\left(T_{1 \mathrm{f}}^{-1}\right)$ is the relaxation rate of the solvent molecules without polymer, and $\rho$ is determined experimentally. (See Figure 1). In terms of a simple two-state model, ${ }^{5} \rho$ can also be expressed as in eq 2.

$$
\rho=\phi\left\{\left(T_{1 \mathrm{a}}^{-1}\right) /\left(T_{1 \mathrm{f}}^{-1}\right)-1\right\}
$$

where $\left(T_{1 \mathrm{a}}^{-1}\right)$ is the relaxation rate of the adsorbed molecules on polymer chains and $\phi$ is the frac- tion of the molecules adsorbed.

Plots of $\rho$ for benzene and methanol vs. mole fraction of methanol are shown in Figure 2. The value of $\rho$ of benzene at the minimum is about 0.1 for both tactic PMMA solutions, which is smaller by a factor of 3 than the values at the high and low concentration of benzene. Considering that the ratio, $\left(T_{1 \mathrm{a}}^{-1}\right) /\left(T_{1 \mathrm{f}}^{-1}\right)$, is almost constant over the entire solvent composition range, $\phi$ would become small at this composition. On the other hand, if $\phi$ is constant, the ratio would become small. In any case, the interaction of benzene with PMMA evidently becomes weak at this composition. A maximum $\rho=0.7$ for the hydroxyl proton is observed near this composition. This means that the benzene molecules are desorbed from PMMA, and the methanol molecules are adsorbed inversely. The same phenomenon has been also observed by several authors through the thermodynamic measurements on ternary systems. ${ }^{2,3}$

$\mathrm{Liu}^{6}$ has measured $T_{1}$ of solvent molecules in polymer solutions, and has shown that the relaxation rate of good solvent is affected more pronouncedly than that of poor solvent. However, he has not experimented over the wide range of solvent composition.

It is interesting to note that the preferential adsorption influences the microscopic interactions of the solvent molecules as well as the equilibrium thermodynamic properties of polymer solution, and the precipitant (methanol) could adsorb on the polymer chain to the degree of affecting the spin-lattice relaxation. It is expected that the hydroxyl group plays an important role in the preferential adsorption.

\section{REFERENCES}

1. A. Dondos and H. Benoît, Makromol Chem., 133, 119 (1970).

2. A. Zǔvny̌, J. Pouchly̌, and K. Sǒlc, Collect. Czech. Chem. Commun., 32, 2753 (1967).

3. J. M. G. Cowie, R. Dey, and J. T. McCrindle, Polymer J., 2, 88 (1971).

4. K. Sato and A. Nishioka, J. Polym. Sci., Part $A-2$, in press.

5. J. R. Zimmerman and W. E. Brittin, J. Phys. Chem., 61, 1328 (1957).

6. K. J. Liu, J. Polym. Sci., Part A-2, 5, 697 (1967). 